\title{
An ultrastructural analysis of platelets, erythrocytes, white blood cells, and fibrin network in systemic lupus erythematosus
}

\author{
Etheresia Pretorius • Jenny du Plooy • \\ Prashilla Soma $\cdot$ Armen Yuri Gasparyan
}

\begin{abstract}
The study suggests that patients with systemic lupus erythematosus (SLE) present with distinct inflammatory ultrastructural changes such as platelets blebbing, generation of platelet-derived microparticles, spontaneous formation of massive fibrin network and fusion of the erythrocytes membranes. Lupoid platelets actively interact with other inflammatory cells, particularly with white blood cells (WBCs), and the massive fibrin network facilitates such an interaction. It is possible that the concerted actions of platelets, erythrocytes and $\mathrm{WBC}$, caught in the inflammatory fibrin network, predispose to pro-thrombotic states in patients with SLE.
\end{abstract}

Keywords Systemic lupus erythematosus - Platelet function - Erythrocytes - White blood cells · Fibrin . Electron microscopy

\section{Introduction}

Systemic lupus erythematosus (SLE) is a chronic, autoimmune disease that can affect any organ system as a result of $\mathrm{T}$ - and B-lymphocytes cooperation and related production of cytotoxic autoantibodies, immune complexes and pro-

E. Pretorius · J. du Plooy · P. Soma

Department of Physiology, Faculty of Health Sciences,

University of Pretoria, Arcadia 0007, South Africa

e-mail: resia.pretorius@up.ac.za

\section{A. Y. Gasparyan ( $\square)$}

Department of Rheumatology, Clinical Research Unit, Dudley Group NHS Foundation Trust (A Teaching Trust of the University of Birmingham, UK), Russells Hall Hospital, Dudley DY1 2HQ, UK

e-mail: a.gasparyan@gmail.com; armen.gasparyan@dgh.nhs.uk inflammatory cytokines [1, 2]. Systemic deposition of immune complexes, activation of the complement components $\mathrm{C} 1 \mathrm{q}$ and $\mathrm{C} 4$ and overproduction of antiphospholipid antibodies are the major triggers of the lupoid cell formation and immune inflammation $[1,3]$. The interaction of immune inflammation with thrombogenesis, in turn, is the basis of the highly prevalent lupoid vascular events $[4,5]$.

Over the past few years, a range of cellular interactions has been implicated in thrombotic vascular events in SLE, with the platelet distinguished as a key element and a target for anti-thrombotic agents [6-8]. Isolated changes in circulating platelet counts (thrombocytopenia) and shape (enlarged volume) have been reported in patients with SLE suffering from arterial and venous thromboses [9]. However, there has not been any report exploring potential links of platelet function changes with other blood cells involvement in lupoid thrombosis.

A recent study pointed out that the shifts in the erythrocyte membrane deformability and enhanced aggregation can contribute to the disturbances in microcirculation and thrombosis in SLE [10]. It was also shown that an elevated level of plasma fibrinogen is a factor behind the enhanced erythrocyte aggregation [10], whereas the deposition of C3 and $\mathrm{C} 4$ complement fragments on the erythrocyte membranes is a link between decreased membrane deformability, tissue hypoxia and SLE-specific organ damage [11].

Another major target of (auto)immune inflammation in SLE is the white blood cell (WBC). Biomarkers of lupoid activity, such as double-stranded DNA and erythrocyte sedimentation rates, correlate with an increased apoptosis of neutrophils [12]. Though the role of neutrophils in thrombosis due to SLE remains obscure, the emerging evidence suggests that antiphospholipid antibodies interacting with neutrophilic biomarkers, such as interleukin-8 and L-selectin, may lead to the thrombogenesis [13]. 
In the current study, we aimed to explore potential mechanisms of platelets interaction with erythrocytes and WBCs in SLE using descriptive analysis of the cellular ultrastructural changes.

\section{Materials and methods}

Blood samples were obtained from healthy subjects aged between 20 and 25 years, both males and females, who did not have any chronic disorders, did not smoke and did not use any medication. The micrographs of fibrin fibres formed in plasma of healthy subjects were compared to our database of thousands of micrographs and found to be comparable.

Citrated blood samples were also obtained from six patients with SLE. These patients were enroled in the study during their admission to the Steve Bio Academic Hospital (Pretoria, South Africa). Three of the six patients were diagnosed previously with lupus nephritis and were all positive for double-stranded DNA. The reports of two patient's ANA results were not available, and the sixth patient was ANA positive. None of the patients had a history of thromboses. Ethical approval was obtained from the University of Pretoria Human Ethics committee, and this study conforms to the principles of the Declaration of Helsinki.
Platelet-rich plasma (PRP) and whole blood (WB)

Smears were prepared on a glass cover slip from PRP as well as WB and incubated at $37{ }^{\circ} \mathrm{C}$ for $5 \mathrm{~min}$ and then immersed in $0.075 \mathrm{M}$ sodium phosphate buffer ( $\mathrm{pH}$ 7.4) and finally placed on a shaker for $2 \mathrm{~min}$.

\section{Extensive fibrin fibre creation}

Ten microlitre of PRP was placed directly on a glass cover slip and immediately mixed with $10 \mu \mathrm{l}$ of thrombin $(10 \mathrm{U} / \mathrm{ml})$ to create an expansive fibrin fibre network. The cover slips with prepared networks were then incubated at room temperature for $5 \mathrm{~min}$ followed by the immersion in $0.075 \mathrm{M}$ sodium phosphate buffer ( $\mathrm{pH}$ 7.4) and finally placed on a shaker for $2 \mathrm{~min}$.

\section{Preparation of buffy layer}

Citrate blood was centrifuged for 20 min to create a buffy layer. $10 \mu \mathrm{l}$ of buffy was placed directly on a glass cover slip and immediately mixed with $10 \mu \mathrm{l}$ of thrombin $(10 \mathrm{U} / \mathrm{ml})$. This was done to investigate the interaction between erythrocytes and WBCs, as well as platelets and fibrin fibres.

All smears were fixed in $2.5 \%$ glutaraldehyde/formaldehyde in PBS solution with a $\mathrm{pH}$ of 7.4 for $30 \mathrm{~min}$,
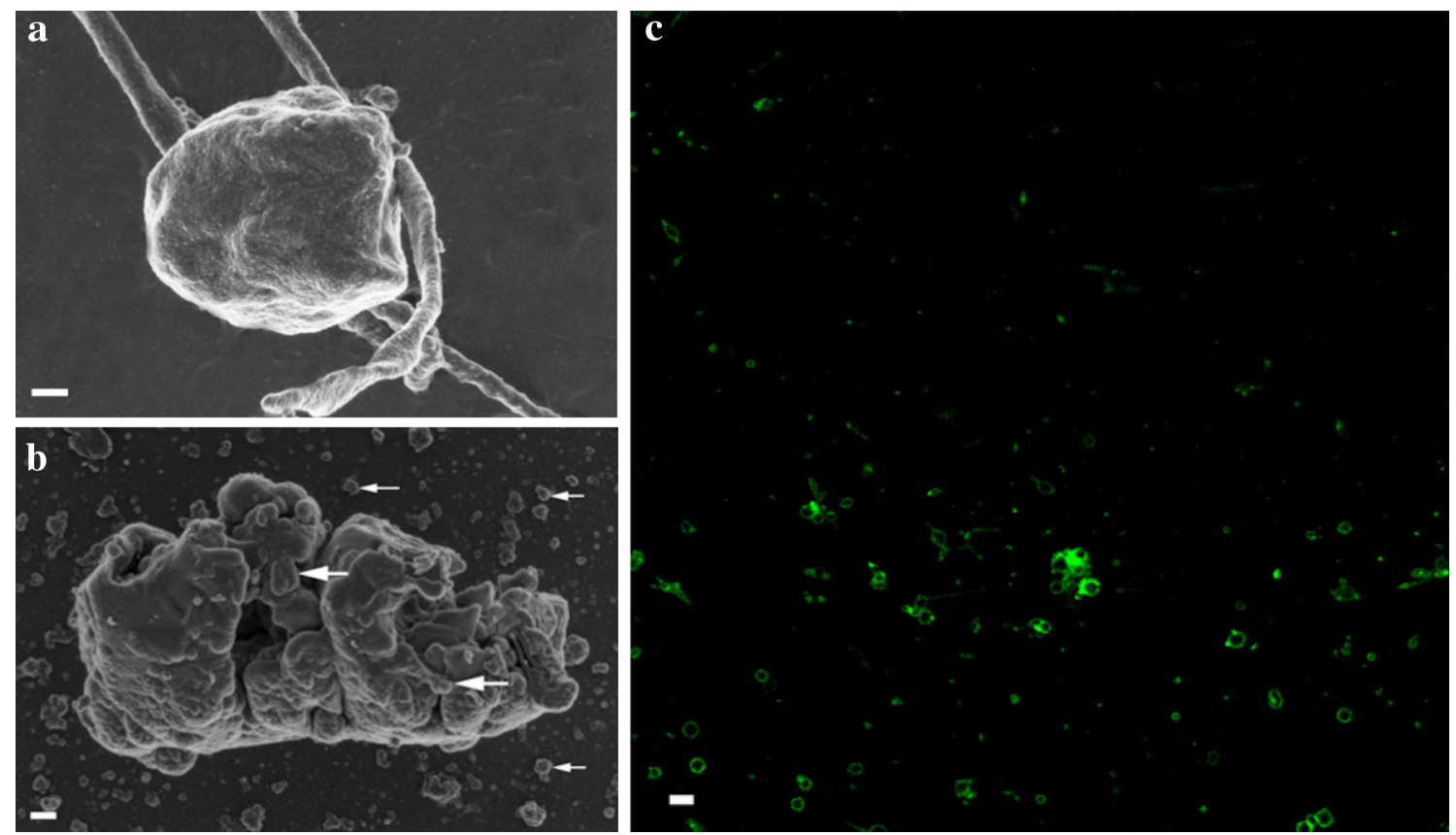

Fig. 1 a Platelet of a healthy subject with smooth membrane and pseudopodia. Scale $1 \mu \mathrm{m}$. b Platelet-rich plasma smear from a lupus patient showing a platelet with membrane blebbing (thick, white arrows), with shedded microparticles around the platelet (thin, white

arrows). Scale $200 \mathrm{~nm}$. c Confocal microscopy of PRP showing platelets and microparticles, confirming platelet origin of microparticles. Scale $5 \mu \mathrm{m}$ 
followed by rinsing $3 \times$ in phosphate buffer for $5 \mathrm{~min}$ before being fixed for 30 min with $1 \%$ osmium tetraoxide (OsO4). The samples were again rinsed $3 \times$ with PBS for 5 min and were dehydrated serially in 30,50, 70, $90 \%$ and three times with $100 \%$ ethanol. The material was critical point dried, mounted and coated with carbon. A Zeiss ULTRA plus FEG-SEM with InLens capabilities were used to study the surface morphology of platelets, and micrographs were taken $1 \mathrm{kV}$. This instrument is located in the Microscopy and Microanalysis Unit of the University of Pretoria, Pretoria, South Africa.

\section{Confocal analysis}

The remaining PRP was transferred to an epindorf and centrifuged for $8 \mathrm{~min}$ to obtain a platelet pellet. The pellet was resuspended in $10 \mu \mathrm{l}$ of the residual PRP using a vortex. $20 \mu \mathrm{l}$ of CD41-FITC (for platelet morphology and for identifying the presence of microparticles originating from platelets) was added to the sample and incubated at room temperature in the dark for $20 \mathrm{~min} .5 \mu \mathrm{l}$ of the sample was mounted on a glass slide and covered with a
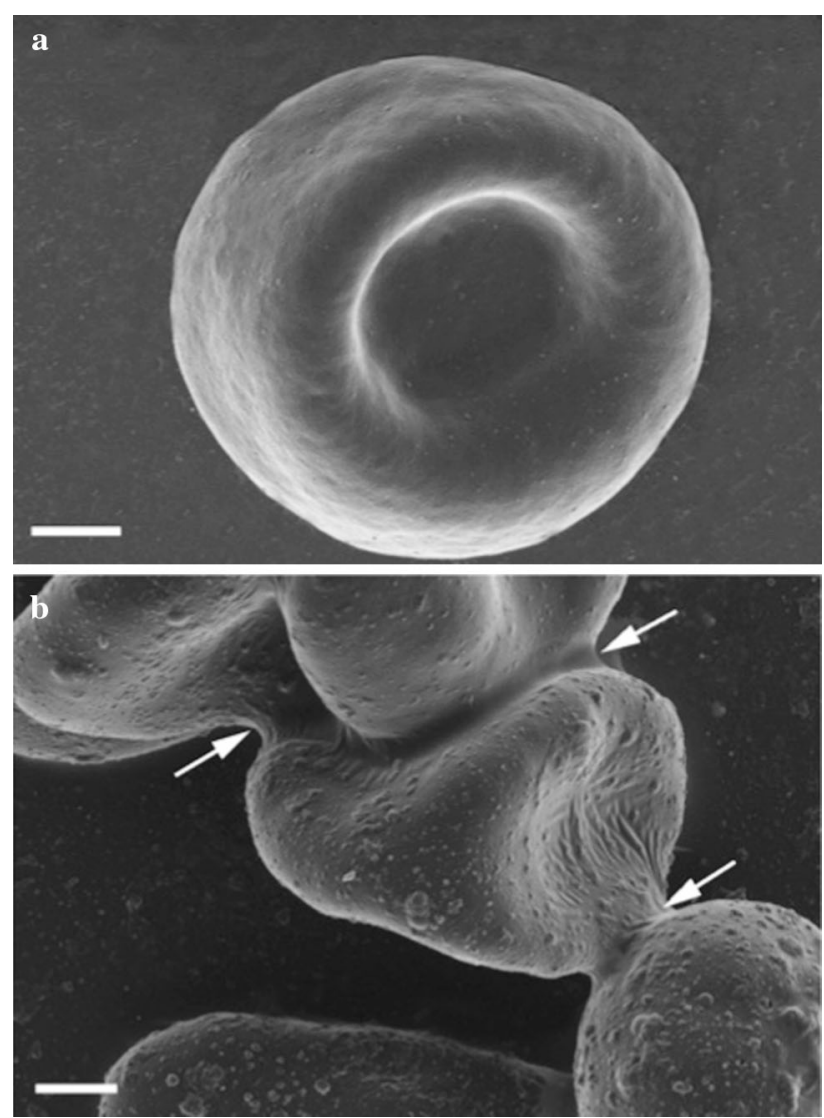

Fig. 2 a Whole blood smear from a healthy subject showing a typical erythrocyte. Scale $1 \mu \mathrm{m}$. b Whole blood smear from a lupus patient showing fused erythrocytes with irregular membrane surfaces (white arrows). Scale $1 \mu \mathrm{m}$ coverslip. The sample was viewed using a Zeiss LSM 510 META confocal microscope with a Plan-Apochromat $63 \times 1$ 1.4 Oil DIC objective. The BP 505-550 filter was used at wavelength $488 \mathrm{~nm}$.

\section{Results}

Platelet ultrastructure from healthy subjects shows the typical bulbous platelet morphology, with smooth platelet membranes and a few pseudopodia on the surface (Fig. 1a). In all SLE patients, the platelet morphology is changed. Membrane blebbing is prevalent on the whole platelet, and these blebs break off to form microparticles visible throughout the smear (Fig. 1b). In order to establish the origin of these microparticles, confocal microscopy was performed using a specific membrane confocal probe (CD41-FITC). This probe proved that the microparticles originate from platelets (Fig. 1c).

We compared the ultrastructure of erythrocytes from a healthy subject (Fig. 2a) and patients with SLE (Fig. 2b).
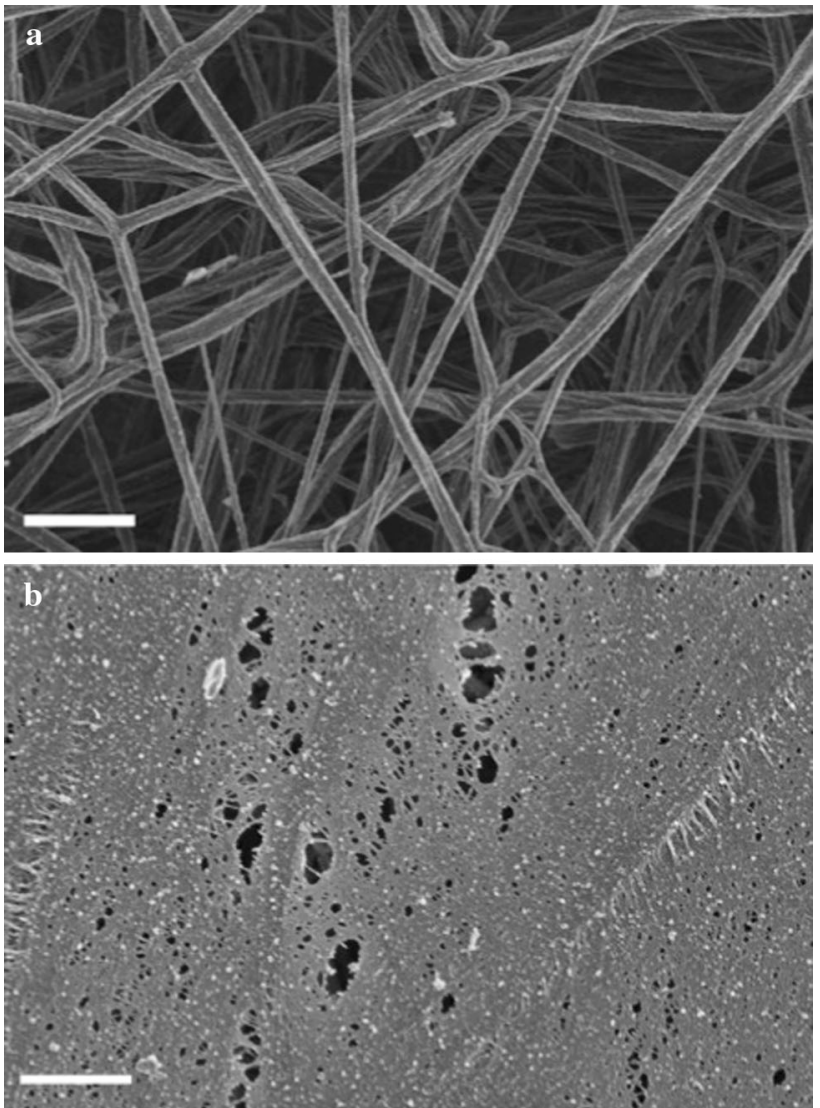

Fig. 3 a Expansive fibrin network created with the addition of thrombin to PRP of a healthy subject. Individual fibrin fibres are visible. Scale $1 \mu \mathrm{m}$. b Expansive fibrin network created with the addition of thrombin to PRP from a lupus patient, showing fibrin fibres coagulated into dense-matted deposits. Scale $1 \mu \mathrm{m}$ 


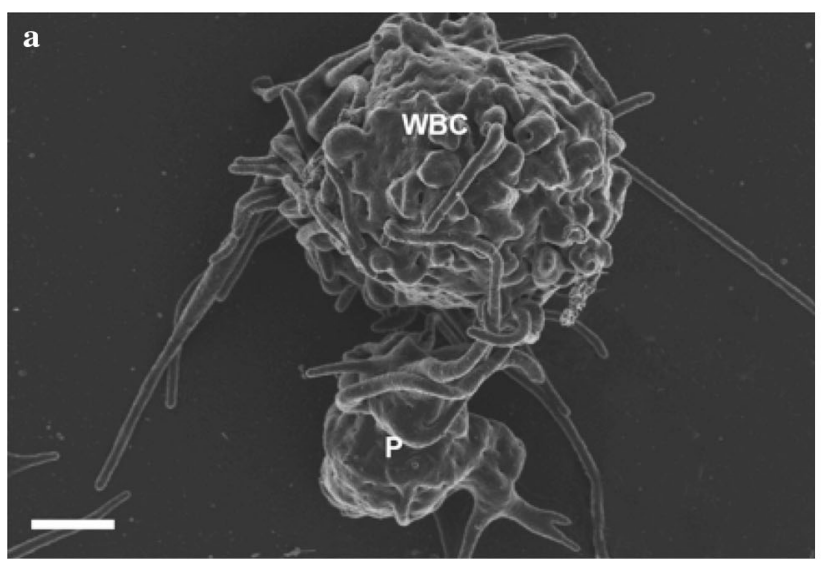

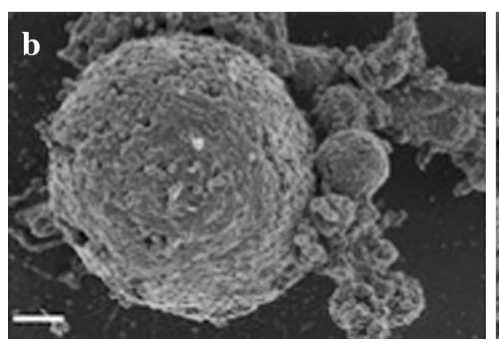

Fig. 4 a Buffy layer smear from a healthy subject showing white blood cell $(W B C)$ and platelet $(P)$ interactions. Scale $1 \mu \mathrm{m}$. b Plate showing buffy layer smear from a lupus patient showing white blood

We found that erythrocytes from lupus patients have fused membranes. This is the first description of fused erythrocytes in SLE.

Expansive fibrin networks can only be created when thrombin is added to PRP. Such a network consists of a netted fibrin fibre layer, where individual fibres are visible (Fig. 3a). In SLE, the fibrin network is altered, with densematted fibrin deposits and invisible individual fibrils (Fig. 3b).

Buffy layer SEM of controls also showed interactions with WBCs, platelets and fibrin. This is depicted in Fig. 4a, b.

\section{Discussion}

Inflammatory rheumatic conditions, particularly SLE, rheumatoid arthritis, Behcet disease, are established clinical models of arterial and venous thromboses linked with dysfunction of platelets and some other blood cells [14-16].

Electron microscope scanning revealed changes of the structure of platelets and erythrocytes exposed to inflammatory microenvironment in SLE. As was shown in our previous study on rheumatoid arthritis, the same microenvironment is responsible for the transformation of the fibrin network from a regular net of fibrils in healthy subjects to an amorphous fibrin deposit [17]. It is possible that inflammatory cellular elements, caught by the transformed cell $(W B C)$ and platelet $(P)$ interactions. Arrows interactions between WBCs and P. Scale $1 \mu \mathrm{m}$

network of fibrin fibres, trigger the firm clot formation resistant to anti-thrombotic agents. Our current observation confirms that lupus patients present with the amorphous fibrin network, which is formed in response to the exposure to thrombin. Moreover, the study suggests that lupoid platelets are prone to their membrane blebbing, which facilitates the generation of highly thrombogenic microparticles [18], known to contribute to lupus nephritis and hypertension [19].

During the platelet activation, microparticles and exosomes are produced and enter the blood circulation. Platelet-derived microparticles express several membrane proteins: glycoprotein (GP)Ib (CD42b), platelet endothelium adhesion molecule-1 (PECAM-1, CD31), GPIIb-IIIa, P-selectin (CD62P), CD63, CD41 and CD61 [20]. CD41 is a highly specific marker for platelet-derived microparticles. In our study, by tagging microparticles with CD41-FITC agent we confirmed that these are predominantly platelet-derived cellular fragments.

For the first time, we observed the structural changes of erythrocytes' membranes in SLE in the form of fusing with each other (Fig. 2b). Our previous studies suggested that erythrocytes are active players in pro-thrombotic and inflammatory disease states, particularly in ischaemic stroke [21]. Current study adds that lupoid erythrocytes are fused and clumped, which may enhance their aggregation, contribute to the disturbances in microcirculation and predispose to vascular events [10]. 
We also found interactions between platelets and WBCs of patients with SLE. These interactions are strongly supported by the transformed fibrin fibrils. Platelets and WBCs function in a close proximity in healthy subjects and in patients with SLE. However, in the former case, cellular membranes are untouched, platelets extend few pseudopodia towards WBCs, and no fibrin fibres are visible (Fig. 4a), whereas lupoid platelets membrane is blebbed, and the cellular interactions are mediated by spontaneously generated fibrin fibrils (Fig. 4b).

Acknowledgments The authors would like to thank Janette Bester and Natasha Vermeulen for the preparation of the SEM samples.

Conflict of interest None declared.

\section{References}

1. Tsokos GC (2011) Systemic lupus erythematosus. N Engl J Med 365(22):2110-2121. doi:10.1056/NEJMra1100359

2. Swadzba J, Iwaniec T, Musial J (2011) Increased level of tumor necrosis factor- $\alpha$ in patients with antiphospholipid syndrome: marker not only of inflammation but also of the prothrombotic state. Rheumatol Int 31(3):307-313. doi:10.1007/s00296009-1314-8

3. Bertsias GK, Salmon JE, Boumpas DT (2010) Therapeutic opportunities in systemic lupus erythematosus: state of the art and prospects for the new decade. Ann Rheum Dis 69(9):1603-1611. doi:10.1136/ard.2010.135186

4. Gasparyan AY, Stavropoulos-Kalinoglou A, Mikhailidis DP, Toms TE, Douglas KM, Kitas GD (2010) The rationale for comparative studies of accelerated atherosclerosis in rheumatic diseases. Curr Vasc Pharmacol 8(4):437-449. doi:10.2174/ 157016110791330852

5. Mashhadi MA, Bari Z (2011) Thrombotic thrombocytopenic purpura and deep vein thrombosis as the presenting manifestations of systemic lupus erythematosus: a case report and review of literature. J Res Med Sci 16(8):1082-1088

6. Boilard E, Blanco P, Nigrovic PA (2012) Platelets: active players in the pathogenesis of arthritis and SLE. Nat Rev Rheumatol 8(9):534-542. doi:10.1038/nrrheum.2012.118

7. Satoh T, Tanaka Y, Okazaki Y, Kaburaki J, Ikeda Y, Kuwana M (2012) Heparin-dependent and -independent anti-platelet factor 4 autoantibodies in patients with systemic lupus erythematosus. Rheumatology (Oxford) 51(9):1721-1728. doi:10.1093/ rheumatology/kes145

8. Hrycek A, Cieślik P (2012) Annexin A5 and anti-annexin antibodies in patients with systemic lupus erythematosus. Rheumatol Int 32(5):1335-1342. doi:10.1007/s00296-011-1793-2
9. Gasparyan AY, Ayvazyan L, Mikhailidis DP, Kitas GD (2011) Mean platelet volume: a link between thrombosis and inflammation? Curr Pharm Des 17(1):47-58. doi:10.2174/13816121 1795049804

10. Spengler MI, Svetaz MJ, Leroux MB, Bertoluzzo SM, Carrara P, Van Isseldyk F, Petrelli D, Parente FM, Bosch P (2011) Erythrocyte aggregation in patients with systemic lupus erythematosus. Clin Hemorheol Microcirc 47(4):279-285. doi:10.3233/ CH-2011-1409

11. Ghiran IC, Zeidel ML, Shevkoplyas SS, Burns JM, Tsokos GC, Kyttaris VC (2011) Systemic lupus erythematosus serum deposits $\mathrm{C} 4 \mathrm{~d}$ on red blood cells, decreases red blood cell membrane deformability, and promotes nitric oxide production. Arthritis Rheum 63(2):503-512. doi:10.1002/art.30143

12. Midgley A, McLaren Z, Moots RJ, Edwards SW, Beresford MW (2009) The role of neutrophil apoptosis in juvenile-onset systemic lupus erythematosus. Arthritis Rheum 60(8):2390-2401. doi:10.1002/art.24634

13. Gladigau G, Haselmayer P, Scharrer I, Munder M, Prinz N, Lackner K, Schild H, Stein P, Radsak MP (2012) A role for tolllike receptor mediated signals in neutrophils in the pathogenesis of the anti-phospholipid syndrome. PLoS One 7(7):e42176. doi:10.1371/journal.pone.0042176

14. Aksu K, Donmez A, Keser G (2012) Inflammation-induced thrombosis: mechanisms, disease associations and management. Curr Pharm Des 18(11):1478-1493. doi:10.2174/1381612127 99504731

15. Gasparyan AY, Stavropoulos-Kalinoglou A, Mikhailidis DP, Douglas KM, Kitas GD (2011) Platelet function in rheumatoid arthritis: arthritic and cardiovascular implications. Rheumatol Int 31(2):153-164. doi:10.1007/s00296-010-1446-x

16. La Regina M, Gasparyan AY, Orlandini F, Prisco D (2010) Behçet's disease as a model of venous thrombosis. Open Cardiovasc Med J 4:71-77. doi:10.2174/1874192401004020071

17. Pretorius E, Oberholzer HM, van der Spuy WJ, Swanepoel AC, Soma P (2012) Scanning electron microscopy of fibrin networks in rheumatoid arthritis: a qualitative analysis. Rheumatol Int 32(6):1611-1615. doi:10.1007/s00296-011-1805-2

18. Porto I, De Maria GL, Di Vito L, Camaioni C, Gustapane M, Biasucci LM (2011) Microparticles in health and disease: small mediators, large role? Curr Vasc Pharmacol 9(4):490-500. doi:10.2174/157016111796197206

19. Nielsen CT, Østergaard O, Johnsen C, Jacobsen S, Heegaard NH (2011) Distinct features of circulating microparticles and their relationship to clinical manifestations in systemic lupus erythematosus. Arthritis Rheum 63(10):3067-3077. doi:10.1002/art. 30499

20. Piccin A, Murphy WG, Smith OP (2007) Circulating microparticles: pathophysiology and clinical implications. Blood Rev 21(3):157-171. doi:10.1016/j.blre.2006.09.001

21. Lipinski B, Pretorius E, Oberholzer HM, van der Spuy WJ (2012) Interaction of fibrin with red blood cells: the role of iron. Ultrastruct Pathol 36(2):79-84. doi:10.3109/01913123.2011.627491 